ANALYSIS \& PDE Volume $7 \quad$ No. $3 \quad 2014$ MARTIN SACK

SCATTERING FOR A MASSLESS CRITICAL NONLINEAR WAVE EQUATION IN TWO SPACE DIMENSIONS 


\title{
SCATTERING FOR A MASSLESS CRITICAL NONLINEAR WAVE EQUATION IN TWO SPACE DIMENSIONS
}

\author{
MARTIN SACK
}

\begin{abstract}
We prove scattering for a massless wave equation which is critical in two space dimensions. Our method combines conformal inversion with decay estimates from Struwe's previous work on global existence of a similar equation.
\end{abstract}

\section{Introduction}

We study the asymptotic behavior of solutions to the nonlinear wave equation

$$
u_{t t}-\Delta u+u\left(e^{u^{2}}-1-u^{2}\right)=0 \quad \text { on } \mathbb{R} \times \mathbb{R}^{2},
$$

with compactly supported initial data

$$
\left.\left(u, u_{t}\right)\right|_{t=0}=\left(u_{0}, u_{1}\right) \in C_{c}^{\infty}\left(\mathbb{R}^{2}\right) \times C_{c}^{\infty}\left(\mathbb{R}^{2}\right) .
$$

Their initial energy is given by

$$
E_{0}=\frac{1}{2} \int_{\mathbb{R}^{2}}\left(u_{1}^{2}+\left|\nabla u_{0}\right|^{2}+e^{u_{0}^{2}}-1-u_{0}^{2}-\frac{1}{2} u_{0}^{4}\right) d x .
$$

Interest in this equation arises because it lies at the boundary of what one considers an energy-critical equation. For the defocusing nonlinear wave equation with power nonlinearity in dimension $d \geq 3$,

$$
u_{t t}-\Delta u+|u|^{p-2} u=0 \quad \text { on } \mathbb{R} \times \mathbb{R}^{d},
$$

this border is marked by the Sobolev-critical power $p^{*}=2 d /(d-2)$. In the subcritical case $p<p^{*}$ as well as in the critical case $p=p^{*}$ well-posedness in the energy space is known to hold. However, little is known for the supercritical case $p>p^{*}$. In two space dimensions the embedding $H^{1}\left(\mathbb{R}^{2}\right) \subset L^{p}\left(\mathbb{R}^{2}\right)$ for $p<\infty$ renders every power nonlinearity subcritical. However, $H^{1}\left(\mathbb{R}^{2}\right) \nsubseteq L^{\infty}\left(\mathbb{R}^{2}\right)$. Instead, we have the Trudinger-Moser inequality

$$
\sup _{\substack{u \in H_{0}^{1}(\Omega) \\\|\nabla u\|_{L^{2}\left(\mathbb{R}^{2}\right)} \leq 1}} \int_{\Omega} e^{\alpha u^{2}} d x \begin{cases}\leq C|\Omega| & \text { if } \alpha \leq 4 \pi, \\ =\infty & \text { if } \alpha>4 \pi,\end{cases}
$$

This work was supported by SNF project 200021_140467 / 1 .

MSC2010: primary 35L71; secondary 35B40.

Keywords: nonlinear wave equation, energy critical, scattering theory. 
for a smooth bounded domain $\Omega \subset \mathbb{R}^{2}$. Since

$$
\sup _{\substack{u \in H_{0}^{1}(\Omega) \\\|\nabla u\|_{L^{2}\left(\mathbb{R}^{2}\right)=1}^{2}}} \int_{\Omega} e^{\alpha u^{2}} d x=\sup _{\substack{u \in H_{0}^{1}(\Omega) \\\|\nabla u\|_{L^{2}\left(\mathbb{R}^{2}\right)}^{2}=\alpha}} \int_{\Omega} e^{u^{2}} d x,
$$

it seems that well-posedness, for instance of the initial value problem for the equation

$$
u_{t t}-\Delta u+u e^{u^{2}}=0 \quad \text { on } \mathbb{R} \times \mathbb{R}^{2}
$$

may depend on the size of the initial energy

$$
E:=\frac{1}{2} \int_{\mathbb{R}^{2}}\left(u_{1}^{2}+\left|\nabla u_{0}\right|^{2}+e^{u_{0}^{2}}-1\right) d x,
$$

(or, in the case of (1), on the size of $E_{0}$ ).

For small data, global well-posedness for (5) was shown in [Nakamura and Ozawa 1999]. Ibrahim, Majdoub, and Masmoudi [Ibrahim et al. 2006] proved global existence for data with energy $E \leq 2 \pi$, which they define to be (sub)critical. Due to the dispersive nature of (5), they also expected $u$ to decay in time and to scatter towards a solution of the linear Klein-Gordon equation

$$
u_{t t}-\Delta u+u=0
$$

Indeed, together with Nakanishi [Ibrahim et al. 2009], they established scattering for the modified equation

$$
u_{t t}-\Delta u+u\left(e^{u^{2}}-u^{2}\right)=0 \quad \text { on } \mathbb{R} \times \mathbb{R}^{2},
$$

as long as

$$
E_{1}=\frac{1}{2} \int_{\mathbb{R}^{2}}\left(u_{1}^{2}+\left|\nabla u_{0}\right|^{2}+e^{u_{0}^{2}}-1-\frac{1}{2} u_{0}^{4}\right) d x \leq 2 \pi,
$$

leaving open the corresponding questions in the supercritical regime when $E>2 \pi$ or $E_{1}>2 \pi$. Note that we reserve the notation $E$ for the context of (5), while $E_{0}$ and $E_{1}$ refer to equations (1) and (7), respectively.

Surprisingly, Struwe [2013] was able to establish global existence for (5) for arbitrary smooth initial data using only energy estimates.

Here we show that for scattering, too, there is no restriction on the energy, at least when we consider the massless wave equation (1) for radially symmetric initial data. As a consequence of the next result, we consider (1), (5), and (7) to be critical problems only, regardless of the size of the initial energy.

Theorem 1.1. For any solution $u$ to the Cauchy problem (1), (2) with smooth compactly supported radial $\operatorname{data}\left(u_{0}, u_{1}\right), u_{0}(x)=u_{0}(|x|), u_{1}(x)=u_{1}(|x|)$, there exists $\left(v_{0}, v_{1}\right) \in \dot{H}^{1}\left(\mathbb{R}^{2}\right) \times L^{2}\left(\mathbb{R}^{2}\right)$ such that

$$
\left\|\left(u(t)-v(t), \partial_{t} u(t)-\partial_{t} v(t)\right)\right\|_{\dot{H}^{1}\left(\mathbb{R}^{2}\right) \times L^{2}\left(\mathbb{R}^{2}\right)} \rightarrow 0 \quad \text { as } t \rightarrow \infty,
$$

where $v$ is the solution to the linear wave equation

$$
v_{t t}-\Delta v=0
$$

with Cauchy data $\left.\left(v, v_{t}\right)\right|_{t=0}=\left(v_{0}, v_{1}\right)$. 
We assume smooth data. We remark, however, that to our knowledge Struwe's result has not been extended to data in energy space.

To prepare for the proof of Theorem 1.1, we rewrite (1) abstractly as

$$
u_{t t}-\Delta u+N=0,
$$

with the nonlinearity

$$
N(u):=\left(e^{u^{2}}-1-u^{2}\right) u .
$$

The solution to (10) is given by the Duhamel formula

$$
u(t)=\partial_{t} R(t) * u_{0}+R(t) * u_{1}+\int_{0}^{t} R(t-s) * N(u(s)) d s
$$

with $R$ the fundamental solution to (9). In Fourier space it reads

$$
\mathscr{F}(R(t))(\xi)=\frac{\sin (|\xi| t)}{|\xi|} .
$$

From the Duhamel formula (11), we read off how the initial data are propagated. We define

$$
v_{0}:=\mathscr{F}^{-1}\left(\hat{u}_{0}-\int_{0}^{\infty} \frac{\sin (|\xi| s)}{|\xi|} \hat{N}(s) d s\right), \quad v_{1}:=\mathscr{F}^{-1}\left(\hat{u}_{1}+\int_{0}^{\infty} \cos (|\xi| s) \hat{N}(s) d s\right)
$$

as initial data for the linear wave equation, which we understand in the trace sense by energy control (compare [Lions and Magenes 1970]). We call $v$ the solution to the corresponding Cauchy problem. Using the Duhamel formula (11), one calculates

$$
\|u(t)-v(t)\|_{\dot{H}^{1}\left(\mathbb{R}^{2}\right)}=\left\|\int_{t}^{\infty} \frac{\sin (|\xi|(t-s))}{|\xi|} \hat{N}(s) d s\right\|_{\dot{H}^{1}\left(\mathbb{R}^{2}\right)},
$$

and a corresponding expression for the time derivative. To prove scattering, we need to establish convergence of the integrals defining the initial data $\left(v_{0}, v_{1}\right)$ in the norm $\dot{H}^{1} \times L^{2}$. In the following lemma we reduce this problem to a bound on the nonlinearity $N$.

Lemma 1.2. If

$$
\|N\|_{L^{1}\left([0, \infty) ; L^{2}\left(\mathbb{R}^{2}\right)\right)}<\infty
$$

the integrals

$$
\int_{0}^{\infty} \frac{\sin (|\xi| s)}{|\xi|} \hat{N}(s) d s, \quad \int_{0}^{\infty} \cos (|\xi| s) \hat{N}(s) d s
$$

converge in $\dot{H}^{1} \times L^{2}$.

The lemma follows from the equivalences

$$
\|u\|_{\dot{H}^{1}} \simeq\||\xi| \hat{u}\|_{L^{2}}, \quad\|v\|_{L^{2}} \simeq\|\hat{v}\|_{L^{2}} .
$$

Thus, once $N \in L_{t}^{1} L_{x}^{2}$ is established, the assertion of Theorem 1.1 follows from (12). 
In the case of the nonlinear Klein-Gordon equation, we find similar representation formulæ and analogous results with the fundamental solution replaced by

$$
\mathscr{F}(R(t))(\xi)=\frac{\sin (\langle\xi\rangle t)}{\langle\xi\rangle}
$$

where $\langle\xi\rangle=\sqrt{1+|\xi|^{2}}$. Then scattering takes place in the norm $H^{1} \times L^{2}$.

This discussion highlights the significance of leaving out the cubic term in (1). Informally, to ensure

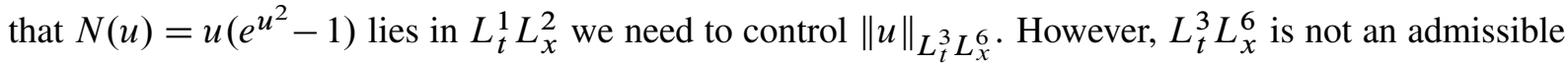
Strichartz norm in two space dimensions. In this respect, we agree with [Ibrahim et al. 2009]. In the course of our argument we will encounter further reasons that justify omission of the cubic term.

Moreover, for large data we restrict our result to the massless equation (1). The reason is that the method of conformal inversion that we employ in Section 3 to control the nonlinearity in this case only seems to work for the massless equation. It is not clear whether a similar control can also be achieved when working in the original coordinates. However, even then, the contribution to the energy from the mass term might spoil the validity of an estimate like Lemma 3.1.

Our work is organized as follows. In Section 2 we derive estimates for the nonlinear term. As a by-product we obtain a scattering result for the massive equation (7) for small data, where we only use standard $L_{t}^{p} L_{x}^{q}$ Strichartz estimates, instead of the more elaborate estimates for Besov spaces used in [Nakamura and Ozawa 1999; Ibrahim et al. 2009].

In Section 3 we prove Theorem 1.1 for large radially symmetric data. In a first step, by applying the method of conformal inversion as in [Grillakis 1990] and adapting the decay estimates from [Struwe 2013], we find a hyperboloid contained inside the support of the solution $u$ such that $\|N\|_{L_{t}^{1} L_{x}^{2}}$ is bounded inside the hyperboloid. For this part of the argument, we need not assume the initial data to be radial. In the final step, we use the radial symmetry of the data to bound $\|N\|_{L_{t}^{1} L_{x}^{2}}$ in the complement of the hyperboloid.

\section{Scattering for small data}

For small data, scattering for (7) was first shown in [Nakamura and Ozawa 1999]. In [Ibrahim et al. 2009], the authors extend the result to include initial data with energy $E_{1} \leq 2 \pi$. Both these works rely on Besov space techniques. In this section, we show scattering for small data via a more direct approach. We assume $u_{0}, u_{1} \in C_{c}^{\infty}\left(\mathbb{R}^{2}\right)$ with $E_{1}$ bounded by an absolute constant $\varepsilon_{0}$ to be determined later.

The modulus of the nonlinearity $|N|=\left(e^{u^{2}}-u^{2}-1\right)|u|$ behaves like $|u|^{5}$ for small values of $|u|$. For large values of $|u|$ the exponential dominates. More precisely, we have the pointwise estimate

$$
|N|=\left|\left(e^{u^{2}}-u^{2}-1\right) u\right|=|u|^{3} \sum_{k=1}^{\infty} \frac{u^{2 k}}{(k+1) !} \leq|u|^{3}\left(e^{u^{2}}-1\right) \leq \begin{cases}|u|^{40 / 9}\left(e^{u^{2}}-1\right) & \text { if }|u| \geq 1, \\ e|u|^{5} & \text { if } 0 \leq|u|<1\end{cases}
$$

By Hölder's inequality,

$$
\left\|u^{40 / 9}\left(e^{u^{2}}-1\right)\right\|_{L_{t}^{1} L_{x}^{2}} \leq\|u\|_{L_{t}^{40 / 9} L_{x}^{20}}^{40 / 9}\left\|e^{u^{2}}-1\right\|_{L_{t}^{\infty} L_{x}^{18 / 5}} .
$$


To control the norm of the exponential term we roughly estimate

$$
\left(e^{u^{2}}-1\right)^{\frac{18}{5}} \leq e^{\frac{18}{5} u^{2}}-1 \leq e^{4 \pi u^{2}}-1 \text {. }
$$

Then we can use a version of the Trudinger-Moser inequality [Ruf 2005]:

$$
\sup _{\|u\|_{L^{2}}+\|\nabla u\|_{L^{2} \leq 1}} \int_{\Omega}\left(e^{4 \pi u^{2}}-1\right) d x \leq C_{\mathrm{TM}}
$$

with a constant $C_{\mathrm{TM}}$ independent of the region $\Omega \subset \mathbb{R}^{2}$. Because of the finite speed of propagation, the support of $u$ stays bounded locally uniformly in time. Since the energy is nonincreasing in time, if $\varepsilon_{0} \leq \frac{1}{2}$, the condition $\|u\|_{L^{2}}+\|\nabla u\|_{L^{2}} \leq 1$ is satisfied for all times. Therefore we may combine (14) with (13) to obtain

$$
\|N\|_{L_{t}^{1}\left([0, T) ; L_{x}^{2}\left(\mathbb{R}^{2}\right)\right)} \leq C_{\mathrm{TM}}\|u\|_{L_{t}^{40 / 9}\left([0, T) ; L_{x}^{20}\left(\mathbb{R}^{2}\right)\right)}^{40 / 9}+e\|u\|_{L_{t}^{5}\left([0, T) ; L_{x}^{10}\left(\mathbb{R}^{2}\right)\right)}^{5}
$$

We have chosen the power $\frac{40}{9}$ for convenience. However, we are not free in our choice, as we want to estimate $u$ in $L_{t}^{q} L_{x}^{r}$ with Strichartz estimates. Wave admissibility [Keel and Tao 1998] demands that

$$
\frac{1}{q}+\frac{1}{2 r} \leq \frac{1}{4}
$$

so we need $q \geq 4$. By Strichartz estimates (as in [Nakanishi and Schlag 2011, Corollary 2.41, Lemma 2.43]),

$$
\begin{aligned}
f(T) & :=\|u\|_{L_{t}^{40 / 9}\left([0, T) ; L_{x}^{20}\left(\mathbb{R}^{2}\right)\right)}+\|u\|_{L_{t}^{5}\left([0, T) ; L_{x}^{10}\left(\mathbb{R}^{2}\right)\right)} \\
& \leq C_{S}\left(\left\|\left(u_{0}, u_{1}\right)\right\|_{H^{1}\left(\mathbb{R}^{2}\right) \times L^{2}\left(\mathbb{R}^{2}\right)}+\|N\|_{L_{t}^{1}\left([0, T) ; L_{x}^{2}\left(\mathbb{R}^{2}\right)\right)}\right)
\end{aligned}
$$

with a constant $C_{S}$ that does not depend on the initial data. Then, by (15) and (16), we have

$$
f(T) \leq C_{S}\left(\left\|\left(u_{0}, u_{1}\right)\right\|_{H^{1}\left(\mathbb{R}^{2}\right) \times L^{2}\left(\mathbb{R}^{2}\right)}+C_{\mathrm{TM}} f(T)^{40 / 9}+e f(T)^{5}\right) .
$$

The function $f(T)$ is continuous and nondecreasing with $f(0)=0$. Therefore there exists a time $T_{0}>0$ such that $f(T) \leq 1$ for $0 \leq T<T_{0}$ and

$$
f(T) \leq C_{S}\left(\left\|\left(u_{0}, u_{1}\right)\right\|_{H^{1}\left(\mathbb{R}^{2}\right) \times L^{2}\left(\mathbb{R}^{2}\right)}+\left(e+C_{\mathrm{TM}}\right) f(T)^{40 / 9}\right)
$$

for all times $T \in\left[0, T_{0}\right)$. Let $A=\min \left\{1, A_{0}\right\}$, where $A_{0}$ satisfies

$$
C_{S}\left(e+C_{\mathrm{TM}}\right)\left(2 A_{0}\right)^{40 / 9}=\frac{1}{2} A_{0} .
$$

Suppose $\left\|\left(u_{0}, u_{1}\right)\right\|_{H^{1}\left(\mathbb{R}^{2}\right) \times L^{2}\left(\mathbb{R}^{2}\right)}<\varepsilon_{0}$, where

$$
C_{S} \varepsilon_{0}=\frac{1}{2} A
$$

Then relation (17) implies $f(T) \leq A$ as long as $f(T) \leq 2 A$. Hence, by continuity, $f\left(T_{0}\right) \leq A$. By the definition of $A$ and continuity again, $T_{0}$ can be arbitrarily extended and the bound $f(T) \leq A$ holds for all times. By (15) we have

$$
\|N\|_{L_{t}^{1}\left([0, \infty) ; L_{x}^{2}\left(\mathbb{R}^{2}\right)\right)} \leq C_{\mathrm{TM}} A^{40 / 9}+e A^{5}<\infty .
$$

Therefore $u$ scatters for $\left\|\left(u_{0}, u_{1}\right)\right\|_{H^{1}\left(\mathbb{R}^{2}\right) \times L^{2}\left(\mathbb{R}^{2}\right)}<\varepsilon_{0}$, and in particular for $E_{1}<\varepsilon_{0}$. 


\section{Scattering for large data}

Conformal inversion. Suppose we are given initial data at time $a>0$. We assume they are compactly supported inside a ball of radius $a / 2$. Because of the finite speed of propagation, the solution is confined within the forward light cone emanating from the origin at time $a / 2$ :

$$
\operatorname{supp} u(t, \cdot) \subset B_{t-a / 2}(0), \quad t \geq a .
$$

We perform a conformal inversion

$$
\Phi:(t, x, u) \mapsto(T, X, U),
$$

as in [Grillakis 1990]; that is, we define

$$
T:=\frac{t}{t^{2}-r^{2}}, \quad X:=\frac{x}{t^{2}-r^{2}}, \quad U:=\Omega^{-\frac{1}{2}} u
$$

with the weight

$$
\Omega:=\frac{1}{t^{2}-r^{2}}=T^{2}-R^{2},
$$

where $r=|x|, R=|X|$. Conformal inversion leaves the structure of the d'Alembert operator invariant [Godin 1994, Lemma 4.2] and

$$
\left(\partial_{T}^{2}-\Delta_{X}\right) U=\Omega^{-\frac{5}{2}}\left(\partial_{t}^{2}-\Delta_{x}\right) u .
$$

In fact, conformal inversion can be regarded as a Kelvin transform of Minkowski space $\left(\mathbb{R}^{1,2}, \eta\right)$ with metric $\eta_{\mu \nu}=\operatorname{diag}(+1,-1,-1)$. This can be seen by writing the transformation as

$$
G: x^{\lambda} \mapsto x^{\lambda}\left(x^{\mu} x^{\nu} \eta_{\mu \nu}\right)^{-1}=x^{\lambda}\langle x, x\rangle_{\eta}^{-1} .
$$

One then calculates the differential,

$d G_{x}(y)=\left.\frac{d}{d t}\right|_{t=0} G(x+t y)=\left.\frac{d}{d t}\right|_{t=0}\left(\frac{x+t y}{\langle x, x\rangle_{\eta}+2 t\langle x, y\rangle_{\eta}+t^{2}\langle y, y\rangle_{\eta}}\right)=\frac{y}{\langle x, x\rangle_{\eta}}-\frac{2 x\langle x, y\rangle_{\eta}}{\langle x, x\rangle_{\eta}^{2}}$, so that $\left\langle\left(d G_{x}\right) y,\left(d G_{x}\right) y\right\rangle_{\eta}=\langle x, x\rangle_{\eta}^{-2}\langle y, y\rangle_{\eta}$ and the differential is a conformal transformation with respect to the metric $\eta$.

In the new variables $T, X$, (1) becomes

$$
\partial_{T}^{2} U-\Delta U+\Omega^{-2} U\left(e^{\Omega U^{2}}-1-\Omega U^{2}\right)=0 .
$$

Note that we changed the direction of time. The transformed function $U$ has support inside the set

$$
\operatorname{supp} U=\left\{(T, X): T+R \leq \frac{2}{a} \text { and } \frac{T}{T^{2}-R^{2}} \geq a \text { and additionally } R \leq T\right\} .
$$

For the following arguments we fix $a$. This is not a restriction. In fact, for any initial data with compact support, we may shift the initial time such that the support of the initial data at the starting time is contained inside our fixed cone. We choose $a=1$. This leads to $\Omega \leq 1$ for $T \leq 1$. 
Energy-flux relation in conformal coordinates. For the remainder of the argument we closely follow [Struwe 2013]. We multiply (18) with $U_{T}$. Then we obtain

$$
\partial_{T} e-\operatorname{div} m=T P
$$

with the scaled energy density

$$
e:=\frac{1}{2}\left(U_{T}^{2}+|\nabla U|^{2}+\Omega^{-3}\left(e^{\Omega U^{2}}-1-\Omega U^{2}-\frac{1}{2} \Omega^{2} U^{4}\right)\right),
$$

the momentum density

$$
m:=U_{T} \nabla U
$$

and the remainder

$$
P:=\Omega^{-4}\left(\Omega U^{2}\left(e^{\Omega U^{2}}-1-\Omega U^{2}\right)-3\left(e^{\Omega U^{2}}-1-\Omega U^{2}-\frac{1}{2} \Omega^{2} U^{4}\right)\right)=U^{8} \sum_{k=0}^{\infty} \frac{\left(\Omega U^{2}\right)^{k}}{(k+4) !}(k+1) \geq 0 .
$$

The power series expansion of $P$ shows that the right-hand side of (19) is positive. Therefore the scaled energy is nonincreasing as we approach the origin. Note that removing the mass term is crucial at this point. Without doing so, we are left with an additional term $-2 \Omega^{-2} U^{2}$ in $P$ that spoils the definite sign of the remainder. Furthermore, the same observation holds for the $u^{3}$-term in the original equation.

For $T_{0}<1$, we integrate (19) over the forward light cone $\{R \leq T\}$ where we truncate by the initial data surface and the support of $U$, that is, we integrate over

$$
K:=\left\{(T, X) \in \operatorname{supp} U, T_{0} \leq T,|X|=R \leq T\right\} .
$$

Its boundary $\partial K$ has four components. The first one is the initial data surface. It contributes the energy $E_{a}$ on the initial data surface. The second is the boundary of the support of $U$ inside $\{R<T\}$. Its contribution vanishes. The third boundary is the mantle of the light cone,

$$
M_{T_{0}}^{1}:=\left\{(T, X): T_{0} \leq T \leq 1,|X|=R=T\right\} .
$$

We write

$$
V(Y):=U(|Y|, Y)
$$

for the restriction of $U$ to the mantle. We call the quantity

$$
\int_{M_{T_{1}}^{T_{2}}} \frac{1}{2}\left(|\nabla V|^{2}+\Omega^{-3}\left(e^{\Omega V^{2}}-1-\Omega V^{2}-\frac{1}{2} \Omega^{2} V^{4}\right)\right) d Y
$$

the flux of $U$ through the mantle $M_{T_{1}}^{T_{2}}$. The last boundary yields the energy in new coordinates:

$$
E\left(T_{0}\right):=\int_{B_{T_{0}}(0)} e d X
$$

Putting everything together, we find

$$
E\left(T_{0}\right)+\frac{1}{\sqrt{2}} \operatorname{Flux}\left(M_{T_{0}}^{1}\right)=E_{a}-\int_{K} P T d X d T .
$$


In particular, we have the energy inequality

$$
E\left(T_{0}\right)+\frac{1}{\sqrt{2}} \operatorname{Flux}\left(M_{T_{0}}^{1}\right) \leq E_{a} .
$$

Therefore the limit $\lim _{T \rightarrow 0} E\left(T, B_{T}(0)\right)$ exists and the flux decays:

$$
\operatorname{Flux}\left(M_{0}^{T}\right):=\sup _{0<S<T} \operatorname{Flux}\left(M_{S}^{T}\right) \rightarrow 0, \quad T \rightarrow 0 .
$$

Moreover, the remainder term $P T$ is bounded by the initial energy

$$
\int_{K} P T d X d T \leq E_{a}
$$

Pointwise estimates for the average on the mantle. We derive pointwise estimates for the spherical averages

$$
\bar{V}=\bar{V}(T)=\frac{1}{2 \pi} \int_{0}^{2 \pi} V\left(e^{i \phi} T\right) d \phi
$$

of $V$, the trace of $U$ on $M_{0}^{T_{0}}$. By Hölder's inequality, for any $0<T \leq T_{1}$,

$$
\begin{aligned}
|\bar{V}(T)| & \leq\left|\bar{V}\left(T_{1}\right)\right|+\int_{T}^{T_{1}}\left|\bar{V}^{\prime}(S)\right| d S \leq\left|\bar{V}\left(T_{1}\right)\right|+\left(\int_{T}^{T_{1}}|\overline{\nabla V}|^{2} S d S \cdot \int_{T}^{T_{1}} \frac{d S}{S}\right)^{\frac{1}{2}} \\
& \leq\left|\bar{V}\left(T_{1}\right)\right|+\pi^{-\frac{1}{2}} \operatorname{Flux}^{\frac{1}{2}}\left(M_{T}^{T_{1}}\right) \log ^{\frac{1}{2}} \frac{T_{1}}{T} .
\end{aligned}
$$

Flux decays towards the origin by (20). So there exists a time $T_{0} \leq 1$ such that, for smaller times $0<T \leq T_{0}$, we have

$$
\operatorname{Flux}^{\frac{1}{2}}\left(M_{T}^{T_{0}}\right) \leq \operatorname{Flux}^{\frac{1}{2}}\left(M_{0}^{T_{0}}\right) \leq \frac{1}{8} .
$$

With this explicit bound on the flux, we can fix a second time $T_{1}, 0<T_{1} \leq T_{0}$, such that $8\left|\bar{V}\left(T_{0}\right)\right| \leq$ $\log ^{1 / 2}(1 / T)$ for $0<T \leq T_{1}$. By $T_{0} \leq 1$ we have $\log \left(T_{0} / T\right) \leq \log (1 / T)$. Therefore,

$$
4|\bar{V}(T)| \leq \log ^{\frac{1}{2}} \frac{1}{T} \quad \text { for all } 0<T \leq T_{1} .
$$

Decay of energy. We introduce polar coordinates $R, \phi$. The energy law (19) becomes

$$
\partial_{T}(R e)-\partial_{R}(R m)=\frac{1}{R} \partial_{\phi}\left(U_{T} U_{\phi}\right)+R T P,
$$

where now

$$
\begin{aligned}
e & :=\frac{1}{2}\left(U_{T}^{2}+U_{R}^{2}+R^{-2} U_{\phi}^{2}+\Omega^{3}\left(e^{\Omega U^{2}}-1-\Omega U^{2}-\frac{1}{2} \Omega^{2} U^{4}\right)\right), \\
m & :=U_{T} U_{R} .
\end{aligned}
$$

We multiply (18) with $X \cdot \nabla U$. Then 


$$
\begin{aligned}
\partial_{T}(X \cdot m)-\operatorname{div}\left(X \cdot \nabla U \nabla U-\frac{X}{2}\left(|\nabla U|^{2}-U_{T}^{2}\right.\right. & \left.\left.+\Omega^{-3}\left(e^{\Omega U^{2}}-1-\Omega U^{2}-\frac{1}{2} \Omega^{2} U^{4}\right)\right)\right) \\
& +U_{T}^{2}-\Omega^{-3}\left(e^{\Omega U^{2}}-1-\Omega U^{2}-\frac{1}{2} \Omega^{2} U^{4}\right)=-R^{2} P .
\end{aligned}
$$

In polar coordinates,

$$
\begin{aligned}
\partial_{T}\left(R^{2} m\right)-\frac{1}{2} \partial_{R}\left(R ^ { 2 } \left(U_{T}^{2}+\right.\right. & \left.\left.U_{R}^{2}-R^{-2} U_{\phi}^{2}-\Omega^{-3}\left(e^{\Omega U^{2}}-1-\Omega U^{2}-\frac{1}{2} \Omega^{2} U^{4}\right)\right)\right) \\
& +R\left(U_{T}^{2}-\Omega^{-3}\left(e^{\Omega U^{2}}-1-\Omega U^{2}-\frac{1}{2} \Omega^{2} U^{4}\right)\right)=\partial_{\phi}\left(U_{R} U_{\phi}\right)-R^{3} P
\end{aligned}
$$

Multiplying (18) with $(U-\bar{V})$, we obtain

$\partial_{T}\left(U_{T}(U-\bar{V})\right)-\operatorname{div}(\nabla U(U-\bar{V}))+|\nabla U|^{2}-U_{T}^{2}+U_{T} \bar{V}_{T}+\Omega^{-2} U(U-\bar{V})\left(e^{\Omega U^{2}}-1-\Omega U^{2}\right)=0$.

Or, again in polar coordinates,

$$
\begin{array}{r}
\partial_{T}\left(R U_{T}(U-\bar{V})\right)-\partial_{R}\left(R U_{R}(U-\bar{V})\right)+R\left(|\nabla U|^{2}-U_{T}^{2}+U_{T} \bar{V}_{T}+\Omega^{-2} U(U-\bar{V})\left(e^{\Omega U^{2}}-1-\Omega U^{2}\right)\right) \\
=\frac{1}{R} \partial_{\phi}\left((U-\bar{V}) U_{\phi}\right)
\end{array}
$$

We rescale the energy identity (24) with $R / T$. Then

$$
\partial_{T}\left(\frac{R^{2}}{T} e\right)-\partial_{R}\left(\frac{R^{2}}{T} m\right)+\frac{R^{2}}{T^{2}} e+\frac{R}{T} m=\partial_{\phi}\left(\frac{1}{T} U_{T} U_{\phi}\right)+R^{2} P .
$$

We divide both (25) and (26) by $T$. Then

$$
\begin{aligned}
\partial_{T}\left(\frac{R^{2}}{T} m\right)- & \frac{1}{2} \partial_{R}\left(\frac{R^{2}}{T}\left(U_{T}^{2}+U_{R}^{2}-R^{-2} U_{\phi}^{2}-\Omega^{-3}\left(e^{\Omega U^{2}}-1-\Omega U^{2}-\frac{1}{2} \Omega^{2} U^{4}\right)\right)\right) \\
& +\frac{R^{2}}{T^{2}} m+\frac{R}{T}\left(U_{T}^{2}-\Omega^{-3}\left(e^{\Omega U^{2}}-1-\Omega U^{2}-\frac{1}{2} \Omega^{2} U^{4}\right)\right)=\partial_{\phi}\left(\frac{1}{T} U_{R} U_{\phi}\right)-\frac{R^{3}}{T} P .
\end{aligned}
$$

and

$$
\begin{aligned}
& \partial_{T}\left(\frac{R}{T} U_{T}(U-\bar{V})\right)-\partial_{R}\left(\frac{R}{T} U_{R}(U-\bar{V})\right) \\
& \quad+\frac{R}{T}\left(|\nabla U|^{2}-U_{T}^{2}+U_{T} \bar{V}_{T}+U_{T} \frac{U-\bar{V}}{T}+\Omega^{-2} U(U-\bar{V})\left(e^{\Omega U^{2}}-1-\Omega U^{2}\right)\right) \\
& =\partial_{T}\left(\frac{R}{T}\left(U_{T}(U-\bar{V})+\frac{(U-\bar{V})^{2}}{2 T}\right)\right)-\partial_{R}\left(\frac{R}{T} U_{R}(U-\bar{V})\right) \\
& \quad+\frac{R}{T}\left(|\nabla U|^{2}-U_{T}^{2}+U_{T} \bar{V}_{T}+V_{T} \frac{U-\bar{V}}{T}+\frac{(U-\bar{V})^{2}}{T^{2}}+\Omega^{-2} U(U-\bar{V})\left(e^{\Omega U^{2}}-1-\Omega U^{2}\right)\right) \\
& =\partial_{\phi}\left(\frac{U-\bar{V}}{R T} U_{\phi}\right) .
\end{aligned}
$$


Adding (27) and (28) with one half of (29) yields

$$
\begin{aligned}
& \partial_{T}\left(\frac{R^{2}}{T}\left(e+m+\frac{1}{2} U_{T} \frac{U-\bar{V}}{R}+\frac{(U-\bar{V})^{2}}{4 T R}\right)\right) \\
& \quad-\partial_{R}\left(\frac{R^{2}}{T}\left(e+m-R^{-2} U_{\phi}^{2}-\Omega^{-3}\left(e^{\Omega U^{2}}-1-\Omega U^{2}-\frac{1}{2} \Omega^{2} U^{4}\right)+U_{R} \frac{U-\bar{V}}{2 R}\right)\right) \\
& \quad+\frac{R}{T}\left(\left(1+\frac{R}{T}\right)(e+m)+\frac{1}{2} U_{T} \bar{V}_{T}+\bar{V}_{T} \frac{U-\bar{V}}{2 T}+\frac{(U-\bar{V})^{2}}{2 T^{2}}\right) \\
& =\partial_{\phi}\left(\frac{1}{T}\left(U_{R}+U_{T}+\frac{U-\bar{V}}{2 R}\right) U_{\phi}\right) \\
& \quad+\frac{R}{T}\left(\frac{3}{2} \Omega^{-3}\left(e^{\Omega U^{2}}-1-\Omega U^{2}-\frac{1}{2} \Omega^{2} U^{4}\right)-\frac{1}{2} \Omega^{-2} U(U-\bar{V})\left(e^{\Omega U^{2}}-1-\Omega U^{2}\right)\right)+R^{2}\left(1-\frac{R}{T}\right) P .
\end{aligned}
$$

Lemma 3.1. For any time $T_{2}$ with $0<T_{2}<T_{1}$, we have

$$
\int_{K^{T_{2}}}\left(\left(1 \pm \frac{R}{T}\right)(e \pm m)+\frac{(U-\bar{V})^{2}}{2 T^{2}}\right) \frac{d X d T}{T} \leq C\left(1+E_{a}+T_{2}^{2} E_{a}^{3}\right)
$$

where $K^{T_{2}}$ is the truncated light cone

$$
K^{T_{2}}:=\left\{(T, X): T \leq T_{2},|X| \leq T\right\} .
$$

Proof. Fix $T_{2}<T_{1}$. We integrate (30) over the truncated cone $K^{T_{2}}$. Then

$$
I_{+}=\int_{K^{T_{2}}}\left(\left(1+\frac{R}{T}\right)(e+m)+\frac{(U-\bar{V})^{2}}{2 T^{2}}\right) \frac{d X d T}{T} \leq \mathrm{II}+\mathrm{IV}+\mathrm{V},
$$

where we label the terms $I_{+}$, II, IV, and V as in the proof of [Struwe 2013, Lemma 3.1]. As shown there, by Poincaré's inequality, we obtain

$$
\mathrm{II} \leq E_{a}, \quad \mathrm{IV} \leq C \operatorname{Flux}\left(M_{0}^{T_{2}}\right) \leq C E_{a} .
$$

The first two terms of our error term

$$
\begin{aligned}
\mathrm{V}=\int_{K^{T_{2}}}\left(-\frac{1}{2} U_{T} \bar{V}_{T}-\bar{V}_{T} \frac{U-\bar{V}}{2 T}+R T\left(1-\frac{R}{T}\right) P\right. \\
\left.\quad+\frac{3}{2} \Omega^{-3}\left(e^{\Omega U^{2}}-1-\Omega U^{2}-\frac{1}{2} \Omega^{2} U^{4}\right)-\frac{1}{2} U(U-\bar{V}) \Omega^{-2}\left(e^{\Omega U^{2}}-1-\Omega U^{2}\right)\right) \frac{d X d T}{T}
\end{aligned}
$$

are the same as in Struwe's work and so, for any $\delta>0$, we have

$$
\left|\int_{K^{T_{2}}}\left(U_{T} \bar{V}_{T}+\bar{V}_{T} \frac{U-\bar{V}}{T}\right) \frac{d X d T}{T}\right| \leq C \delta \int_{0}^{T_{2}} \int_{B_{T / 2}(0)}|\nabla U|^{2} \frac{d X d T}{T}+C \delta I_{+}+C \delta^{-1} \operatorname{Flux}\left(M_{0}^{T_{2}}\right) .
$$

By (21),

$$
\int_{K^{T_{2}}} R\left(1-\frac{R}{T}\right) P d X d T \leq \int_{K^{T_{2}}} T P d X d T \leq E_{a}
$$


For the remaining terms we add and subtract in the spherical averages as defined in (22):

$$
\begin{gathered}
\frac{3}{2} \Omega^{-3}\left(e^{\Omega U^{2}}-1-\Omega U^{2}-\frac{1}{2} \Omega^{2} U^{4}\right)-\frac{1}{2} U(U-\bar{V}) \Omega^{-2}\left(e^{\Omega U^{2}}-1-\Omega U^{2}\right) \\
=\frac{3}{2} \Omega^{-3}\left(e^{\Omega U^{2}}-1-\Omega U^{2}-\frac{1}{2} \Omega^{2} U^{4}-\left(e^{\Omega \bar{V}^{2}}-1-\Omega \bar{V}^{2}-\frac{1}{2} \Omega^{2} \bar{V}^{4}\right)\right) \\
\quad-\frac{1}{2} U(U-\bar{V}) \Omega^{-2}\left(e^{\Omega U^{2}}-1-\Omega U^{2}\right)+\frac{3}{2} \Omega^{-3}\left(e^{\Omega \bar{V}^{2}}-1-\Omega \bar{V}^{2}-\frac{1}{2} \Omega^{2} \bar{V}^{4}\right) \\
=f(U, \bar{V})+\frac{3}{2} \Omega^{-3}\left(e^{\Omega \bar{V}^{2}}-1-\Omega \bar{V}^{2}-\frac{1}{2} \Omega^{2} \bar{V}^{4}\right) .
\end{gathered}
$$

We can compensate for the second term with the pointwise bound from (23):

$$
\begin{aligned}
\frac{3}{2} \Omega^{-3}\left(e^{\Omega \bar{V}^{2}}-1-\Omega \bar{V}^{2}-\frac{1}{2} \Omega^{2} \bar{V}^{4}\right) & =\frac{3}{2} \sum_{k=3}^{\infty} \frac{\Omega^{k-3} \bar{V}^{2 k}}{k !} \\
& =\frac{3}{2} \bar{V}^{6} \sum_{k=0}^{\infty} \frac{\left(\Omega \bar{V}^{2}\right)^{k}}{(k+3) !} \leq \frac{3}{2} \bar{V}^{6} e^{\Omega^{2}} \\
& \leq C \log ^{3}\left(\frac{1}{T}\right) \frac{1}{T \frac{1}{16} \Omega} \leq C \log ^{3}\left(\frac{1}{T}\right) \frac{1}{T},
\end{aligned}
$$

where we used $\Omega \leq 1$. Then

$$
\int_{K^{T_{2}}} \log ^{3}\left(\frac{1}{T}\right) \frac{1}{T} \frac{d X d T}{T} \leq C \int_{0}^{T} \log ^{3}\left(\frac{1}{T}\right) d T \leq C<\infty .
$$

In the following, we analyze the nonlinear function $f$ as above by comparing $U(T, X)$ with $\bar{V}(T)$ pointwise in $X$ for a fixed time slice. Recalling that

$$
f(U, \bar{V})=\frac{3}{2} \sum_{k=3}^{\infty} \frac{\Omega^{k-3}\left(U^{2 k}-\bar{V}^{2 k}\right)}{k !}-\frac{1}{2} U(U-\bar{V}) \sum_{k=2}^{\infty} \frac{\Omega^{k-2} U^{2 k}}{k !},
$$

we observe that $f(-U,-\bar{V})=f(U, \bar{V})$. Furthermore, if $U$ and $\bar{V}$ have opposite sign, say $U \geq 0, \bar{V} \leq 0$, then $U(U-\bar{V}) \geq U^{2}$. Comparing coefficients, we see that the second power series dominates the first and $f$ is negative. Therefore, we only need to analyze the case $U, \bar{V}>0$. We distinguish three subcases. (i) If $U \leq \bar{V}$, then

$$
f(U, \bar{V}) \leq \frac{1}{2} \bar{V}^{2} \Omega^{-2}\left(e^{\Omega \bar{V}^{2}}-1-\Omega \bar{V}^{2}\right) \leq \frac{1}{2} \bar{V}^{6} e^{\Omega \bar{V}^{2}},
$$

which we estimate with the bound on $|\bar{V}|$ as above.

(ii) If $\bar{V}<U \leq 4 \bar{V}$, then

$$
f(U, \bar{V}) \leq \frac{3}{2} \Omega^{-3}\left(e^{16 \Omega \bar{V}^{2}}-1-16 \Omega \bar{V}^{2}-\frac{1}{2}(16 \Omega)^{2} \bar{V}^{4}\right) \leq \frac{3}{2} 16^{3} \bar{V}^{6} e^{16 \Omega \bar{V}^{2}} \leq C \log ^{3}\left(\frac{1}{T}\right) \frac{1}{T},
$$

where the factor 4 in (23) together with $\Omega \leq 1$ ensures that the power in $1 / T$ stays smaller than 1 .

(iii) For the remaining case $U>4 \bar{V}$, we write $\bar{V}=\alpha U$, that is, $\alpha<\frac{1}{4}$. Then we analyze the power series

$$
f(U, \bar{V})=\frac{1}{4}\left(U^{6}-\bar{V}^{6}\right)+\frac{3}{2} \sum_{k=4}^{\infty} \frac{\Omega^{k-3}\left(U^{2 k}-\bar{V}^{2 k}\right)}{k !}-\frac{1}{2} U(U-\bar{V}) \sum_{k=2}^{\infty} \frac{\Omega^{k-2} U^{2 k}}{k !} .
$$


For the leading term, we use $\alpha<\frac{1}{4}$ to compare with $(U-\bar{V})^{6}$,

$$
U^{6}-\bar{V}^{6}=U^{6}\left(1-\alpha^{6}\right) \leq C U^{6}(1-\alpha)^{6}=C(U-\bar{V})^{6},
$$

pointwise. Then, by the Poincaré-Sobolev inequality, on each time slice,

$$
\int_{B_{T}(0)} \frac{(U-\bar{V})^{6}}{T} d X \leq \frac{C}{T}\left(\int_{B_{T}(0)}|\nabla U|^{\frac{3}{2}} d X\right)^{6} \leq C T\left(\int_{B_{T}(0)}|\nabla U|^{2} d X\right)^{3} \leq C T E_{a}^{3} .
$$

Integration in time yields a term bounded by $T_{2}^{2} E_{a}^{3}$. The remaining power series is negative, as

$$
\begin{aligned}
\frac{3}{2} \sum_{k=4}^{\infty} \frac{\Omega^{k-3}\left(U^{2 k}-\bar{V}^{2 k}\right)}{k !}- & \frac{1}{2} U(U-\bar{V}) \sum_{k=2}^{\infty} \frac{\Omega^{k-2} U^{2 k}}{k !} \\
& =\frac{3}{2} U^{6} \sum_{k=1}^{\infty} \frac{\left(\Omega U^{2}\right)^{k}\left(1-\alpha^{2(k+3)}\right)}{(k+3) !}-\frac{1}{4}(1-\alpha) U^{6} \sum_{k=0}^{\infty} \frac{\left(\Omega U^{2}\right)^{k}}{(k+2) !} \\
& =U^{6}\left(-\frac{1}{2}(1-\alpha)+\sum_{k=1}^{\infty} \frac{\left(\Omega U^{2}\right)^{k}}{(k+3) !}\left(\frac{1}{2}\left(3\left(1-\alpha^{2(k+3)}\right)-(1-\alpha)(k+3)\right)\right)\right) \\
& \leq 0 .
\end{aligned}
$$

Note that this calculation further motivates the exclusion of $u^{3}$ in the original equation.

Combining, we arrive at the estimate

$$
\mathrm{V} \leq C\left(1+E_{a}+T_{2}^{2} E_{a}^{3}+\delta I_{+}\right)+C \delta \int_{0}^{T_{2}} \int_{B_{T / 2}(0)}|\nabla U|^{2} \frac{d X d T}{T}+C \delta^{-1} \operatorname{Flux}\left(M_{0}^{T_{2}}\right) .
$$

By the energy inequality, $\operatorname{Flux}\left(M_{0}^{T_{2}}\right) \leq E_{a}$. Therefore,

$$
I_{+} \leq C\left(1+E_{a}+T_{2}^{2} E_{a}^{3}+\delta I_{+}+\delta^{-1} E_{a}\right)+C \delta \int_{0}^{T_{2}} \int_{B_{T / 2}(0)}|\nabla U|^{2} \frac{d X d T}{T},
$$

and, in the same fashion,

$$
\begin{aligned}
I_{-} & =\int_{K^{T_{2}}}\left(\left(1-\frac{R}{T}\right)(e-m)+\frac{(U-\bar{V})^{2}}{2 T^{2}}\right) \frac{d X d T}{T} \\
& \leq C\left(1+E_{a}+T_{2}^{2} E_{a}^{3}+\delta I_{+}+\delta^{-1} E_{a}\right)+C \delta \int_{0}^{T_{2}} \int_{B_{T / 2}(0)}|\nabla U|^{2} \frac{d X d T}{T} .
\end{aligned}
$$

We have $|\nabla U|^{2} \leq 2 e=(e+m)+(e-m)$, and hence

$$
\int_{0}^{T_{2}} \int_{B_{T / 2}(0)}|\nabla U|^{2} \frac{d X d T}{T} \leq I_{+}+2 I_{-} .
$$

Choosing $\delta>0$ sufficiently small, we conclude that

$$
I_{+}+I_{-} \leq C\left(1+E_{a}+T_{2}^{2} E_{a}^{3}\right) .
$$


Bound inside a hyperboloid. Recall that $T_{1}$ was fixed to bound $|\bar{V}(T)|$ as in (23), which in turn was crucial for smallness in Lemma 3.1.

For any $\varepsilon>0$, we fix a time $0<T_{\varepsilon}<T_{1}$ such that

$$
\operatorname{Flux}\left(u, M^{T_{\varepsilon}}\right)+\int_{K^{T_{\varepsilon}}}\left(\left(1 \pm \frac{R}{T}\right)(e \pm m)+\frac{(U-\bar{V})^{2}}{T^{2}}\right) \frac{d X d T}{T}<\varepsilon .
$$

In the same fashion as in [Struwe 2013, Lemma 4.3], we obtain:

Lemma 3.2. There exists $\varepsilon>0$ and a constant $C<\infty$ such that, for any $0<T \leq 4^{-1} T_{\varepsilon}$, we have

$$
\int_{K^{T}} e^{4 U^{2}} d X d T \leq C T
$$

The region $\Phi^{-1}\left(K^{T}\right)$ is a hyperboloid. Its asymptote is the cone $\{r=t-1 /(2 T)\}$.

In the following we fix $T \leq 4^{-1} T_{\epsilon}$. Let $t_{0}=1 / T$, the smallest time inside the hyperboloid. Furthermore, we denote $D=\Phi^{-1}\left(K^{T}\right)$.

Using Lemma 3.2, we obtain decay of the nonlinearity in $L_{t}^{2} L_{x}^{2}$ locally in time.

Lemma 3.3. Let $t_{2} \geq t_{1} \geq t_{0}$. Then

$$
\int_{D \cap\left\{t_{1} \leq t \leq t_{2}\right\}}|N(u)|^{2} d x d t \leq C t_{1}^{-2} .
$$

Proof. Inside $D_{t_{1}}^{t_{2}}=D \cap\left\{t_{1} \leq t \leq t_{2}\right\}$ we have $t+r \geq t$ and $t-r \geq 1 /(2 T)$. Therefore, $\Omega \leq C / t_{1}$ with a constant $C$ that is uniform over $D_{t_{1}}^{t_{2}}$. Then we calculate

$$
\begin{aligned}
\int_{D_{t_{1}}^{t_{2}}}\left|u\left(e^{u^{2}}-1-u^{2}\right)\right|^{2} d x d t & =\int_{\Phi\left(D_{t_{1}}^{t_{2}}\right)} \Omega U^{2}\left(e^{\Omega U^{2}}-1-\Omega U^{2}\right)^{2} \Omega^{-3} d X d T \\
& \leq \int_{\Phi\left(D_{t_{1}}^{t_{2}}\right)} \frac{1}{4} \Omega^{2} U^{10} e^{2 \Omega U^{2}} d X d T \leq \frac{C}{t_{1}^{2}} \int_{\Phi\left(D_{t_{1}}^{t_{2}}\right)} e^{3 U^{2}} d X d T \leq C \frac{T}{t_{1}^{2}}
\end{aligned}
$$

We conclude:

Lemma 3.4. Inside $D$ the nonlinearity is bounded in $L_{t}^{1} L_{x}^{2}$, that is,

$$
\int_{t_{0}}^{\infty}\left(\int_{D \cap\left(\{t\} \times \mathbb{R}^{2}\right)}|N|^{2} d x\right)^{\frac{1}{2}} d t<\infty .
$$

Proof. Divide $\left[t_{0}, \infty\right)$ into intervals $I_{n}=\left[t_{0} 2^{n}, t_{0} 2^{n+1}\right)$. Then, by Hölder's inequality and Lemma 3.3,

$$
\begin{aligned}
\int_{t_{0}}^{\infty}\left(\int_{D \cap\left(\{t\} \times \mathbb{R}^{2}\right)}|N|^{2} d x\right)^{\frac{1}{2}} d t & =\sum_{n=0}^{\infty} \int_{I_{n}}\left(\int_{D \cap\left(\{t\} \times \mathbb{R}^{2}\right)}|N|^{2} d x\right)^{\frac{1}{2}} d t \\
& \leq \sum_{n=0}^{\infty}\left(t_{0} 2^{n}\right)^{\frac{1}{2}}\left(\int_{I_{n}} \int_{D \cap\left(\{t\} \times \mathbb{R}^{2}\right)}|N|^{2} d x d t\right)^{\frac{1}{2}} \\
& \leq \sum_{n=0}^{\infty} C t_{0}^{-\frac{1}{2}} 2^{-n / 2}<\infty
\end{aligned}
$$


The case of radial data. In the previous section we have obtained control of the nonlinearity inside a hyperboloid $\Phi^{-1}\left(K^{T}\right)$, where $T \leq 4^{-1} T_{\epsilon}$. Let $t_{0}=1 / T$, the smallest time in the hyperboloid. Now fix $T$ and choose $d>1 /(2 T)$. Let

$$
A_{t_{1}}=\left\{(t, x): t \geq t_{1}, t-d \leq|x| \leq t\right\} .
$$

Then there exists a time $t_{1} \geq t_{0}$ such that

$$
\left\{(t, x): t \geq t_{1},|x| \leq t\right\} \subset\left(\Phi^{-1}\left(K^{T}\right) \cap\left\{(t, x): t \geq t_{1}\right\}\right) \cup A_{t_{1}},
$$

that is, the thinned cone $A_{t_{1}}$ covers the region where we have not yet obtained control over the nonlinearity.

In the following, we will restrict ourselves to the case of radial solutions. We will show that we can bound the nonlinearity inside $A_{t_{1}}$ in $L_{t}^{1} L_{x}^{2}$.

In the case of radially symmetric data, we employ the following bound. Let $t>t_{1}$ fixed, $t-d \leq r \leq t$. Recall that $u$ is compactly supported within $B_{t}(0)$. Then

$$
\begin{aligned}
|u(t, r)| & \leq \int_{r}^{t}\left|\partial_{s} u(t, s)\right| d s \leq \int_{t-d}^{t}\left|\partial_{s} u(t, s)\right| d s \\
& \leq\left(\int_{t-d}^{t}\left|\partial_{s} u(t, s)\right|^{2} s d s\right)^{\frac{1}{2}}\left(\int_{t-d}^{t} \frac{1}{s} d s\right)^{\frac{1}{2}} \leq C E^{\frac{1}{2}}\left(\log \frac{t}{t-d}\right)^{\frac{1}{2}} .
\end{aligned}
$$

Therefore there exists $t_{2} \geq t_{1}$ such that $|u(t, r)| \leq \frac{C}{t^{1 / 2}}$ for all $t \geq t_{2}$, with a constant $C$ independent of $t \geq t_{2}$.

Lemma 3.5. Let $t_{2}$ be as above. Then $N$ is bounded in $L_{t}^{1} L_{x}^{2}$ inside $A_{t_{2}}$.

Proof. Again we estimate

$$
|N(u)|=|u|\left(e^{u^{2}}-1-u^{2}\right) \leq \frac{1}{2}|u|^{5} e^{u^{2}}
$$

pointwise. Then

Therefore,

$$
\int_{B_{t}(0) \backslash B_{t-d}(0)} u^{10} e^{2 u^{2}} d x \leq C t \cdot t^{-5}=C t^{-4}
$$

$$
\int_{t_{2}}^{\infty}\left(\int_{B_{t}(0) \backslash B_{t-d}(0)} u^{10} e^{2 u^{2}} d x\right)^{\frac{1}{2}} d t \leq C \int_{t_{2}}^{\infty} t^{-2} d t<\infty .
$$

Combining Lemmas 3.3 and 3.5, we obtain $\|N\|_{L^{1}\left(\left[t_{2}, \infty\right) ; L^{2}\left(\mathbb{R}^{2}\right)\right)}<\infty$. Using Lemma 1.2, we conclude the proof of Theorem 1.1

\section{References}

[Godin 1994] P. Godin, "Global sound waves for quasilinear second order wave equations", Math. Ann. 298:3 (1994), 497-531. MR 95f:35156 Zbl 0790.35071

[Grillakis 1990] M. G. Grillakis, "Regularity and asymptotic behaviour of the wave equation with a critical nonlinearity", Ann. of Math. (2) 132:3 (1990), 485-509. MR 92c:35080 Zbl 0736.35067 
[Ibrahim et al. 2006] S. Ibrahim, M. Majdoub, and N. Masmoudi, "Global solutions for a semilinear, two-dimensional KleinGordon equation with exponential-type nonlinearity”, Comm. Pure Appl. Math. 59:11 (2006), 1639-1658. MR 2007h:35229 Zbl 1117.35049

[Ibrahim et al. 2009] S. Ibrahim, M. Majdoub, N. Masmoudi, and K. Nakanishi, "Scattering for the two-dimensional energycritical wave equation", Duke Math. J. 150:2 (2009), 287-329. MR 2010k:35313 Zbl 1206.35175

[Keel and Tao 1998] M. Keel and T. Tao, "Endpoint Strichartz estimates”, Amer. J. Math. 120:5 (1998), 955-980. MR 2000d: 35018 Zbl 0922.35028

[Lions and Magenes 1970] J.-L. Lions and E. Magenes, Problèmes aux limites non homogènes et applications, vol. 3, Travaux et Recherches Mathématiques 20, Dunod, Paris, 1970. Translated as Non-homogeneous boundary value problems and applications, vol. 3, Grundlehren der Mathematischen Wissenschaften 183, Springer, New York, 1973. MR 45 \#975 Zbl 0197.06701

[Nakamura and Ozawa 1999] M. Nakamura and T. Ozawa, "Global solutions in the critical Sobolev space for the wave equations with nonlinearity of exponential growth", Math. Z. 231:3 (1999), 479-487. MR 2001b:35216 Zbl 0931.35107

[Nakanishi and Schlag 2011] K. Nakanishi and W. Schlag, Invariant manifolds and dispersive Hamiltonian evolution equations, European Mathematical Society, Zürich, 2011. MR 2012m:37120 Zbl 1235.37002

[Ruf 2005] B. Ruf, “A sharp Trudinger-Moser type inequality for unbounded domains in $\mathbb{R}^{2}$ ”, J. Funct. Anal. 219:2 (2005), 340-367. MR 2005k:46082 Zbl 1119.46033

[Struwe 2013] M. Struwe, "The critical nonlinear wave equation in two space dimensions", J. Eur. Math. Soc. 15:5 (2013), 1805-1823. MR 3082244 Zbl 1282.35245

Received 4 Jun 2013. Revised 14 Feb 2014. Accepted 11 Apr 2014.

MARTIN SACK: sackm@phys.ethz.ch

Department of Mathematics, ETH Zürich, CH-8092 Zürich, Switzerland 


\title{
Analysis \& PDE
}

\author{
msp.org/apde
}

\section{EDITORS}

EDITOR-IN-CHIEF

\author{
Maciej Zworski \\ zworski@math.berkeley.edu \\ University of California \\ Berkeley, USA
}

BOARD OF EDITORS

Nicolas Burq Université Paris-Sud 11, France

nicolas.burq@math.u-psud.fr

Sun-Yung Alice Chang Princeton University, USA

chang@math.princeton.edu

Michael Christ University of California, Berkeley, USA

mchrist@math.berkeley.edu

Charles Fefferman Princeton University, USA

cf@math.princeton.edu

Ursula Hamenstaedt Universität Bonn, Germany

ursula@math.uni-bonn.de

Vaughan Jones U.C. Berkeley \& Vanderbilt University vaughan.f.jones@vanderbilt.edu

Herbert Koch Universität Bonn, Germany koch@math.uni-bonn.de

Izabella Laba University of British Columbia, Canada ilaba@math.ubc.ca

Gilles Lebeau Université de Nice Sophia Antipolis, France lebeau@unice.fr

László Lempert Purdue University, USA lempert@math.purdue.edu

Richard B. Melrose Massachussets Institute of Technology, USA rbm@math.mit.edu

Frank Merle Université de Cergy-Pontoise, France Frank.Merle@u-cergy.fr

William Minicozzi II Johns Hopkins University, USA minicozz@math.jhu.edu

Werner Müller Universität Bonn, Germany mueller@math.uni-bonn.de
Yuval Peres

Gilles Pisier

Tristan Rivière

Igor Rodnianski

Wilhelm Schlag

Sylvia Serfaty

Yum-Tong Siu

Terence Tao

Michael E. Taylor

Gunther Uhlmann

András Vasy

Dan Virgil Voiculescu

Steven Zelditch
University of California, Berkeley, USA

peres@stat.berkeley.edu

Texas A\&M University, and Paris 6

pisier@math.tamu.edu

ETH, Switzerland

riviere@math.ethz.ch

Princeton University, USA

irod@math.princeton.edu

University of Chicago, USA

schlag@math.uchicago.edu

New York University, USA

serfaty@cims.nyu.edu

Harvard University, USA

siu@math.harvard.edu

University of California, Los Angeles, USA

tao@math.ucla.edu

Univ. of North Carolina, Chapel Hill, USA

met@math.unc.edu

University of Washington, USA

gunther@math.washington.edu

Stanford University, USA

andras@math.stanford.edu

University of California, Berkeley, USA

dvv@math.berkeley.edu

Northwestern University, USA

zelditch@math.northwestern.edu

\section{PRODUCTION}

production@msp.org

Silvio Levy, Scientific Editor

See inside back cover or msp.org/apde for submission instructions.

The subscription price for 2014 is US $\$ 180 /$ year for the electronic version, and $\$ 355 /$ year $(+\$ 50$, if shipping outside the US) for print and electronic. Subscriptions, requests for back issues from the last three years and changes of subscribers address should be sent to MSP.

Analysis \& PDE (ISSN 1948-206X electronic, 2157-5045 printed) at Mathematical Sciences Publishers, 798 Evans Hall \#3840, c/o University of California, Berkeley, CA 94720-3840, is published continuously online. Periodical rate postage paid at Berkeley, CA 94704, and additional mailing offices.

APDE peer review and production are managed by EditFLOW ${ }^{\circledR}$ from Mathematical Sciences Publishers.

PUBLISHED BY

- mathematical sciences publishers

nonprofit scientific publishing

http://msp.org/

(C) 2014 Mathematical Sciences Publishers 


\section{ANALYSIS \& PDE}

\section{Volume $7 \quad$ No. $3 \quad 2014$}

Prescription du spectre de Steklov dans une classe conforme

PIERRE JAMMES

Semilinear geometric optics with boundary amplification

JEAN-Francois COUlOMBEL, OliVier GuÈs and MARK Williams

The 1-harmonic flow with values in a hyperoctant of the $N$-sphere

Lorenzo Giacomelli, Jose M. Mazón and SAlvador Moll

Decomposition rank of $\mathscr{L}$-stable $\mathrm{C}^{*}$-algebras

AARON TIKUISIS and WILHELM WINTER

Scattering for a massless critical nonlinear wave equation in two space dimensions

MARTIN SACK

Large-time blowup for a perturbation of the cubic Szegő equation

HAIYAN XU

A geometric tangential approach to sharp regularity for degenerate evolution equations

EduARdo V. TeIXEIRA and José Miguel UrbanO

The theory of Hahn-meromorphic functions, a holomorphic Fredholm theorem, and its appli- 745 cations

JÖRN MÜLlER and AlEXANDER STROHMAIER 